\title{
Aspectos microestruturais e de resistência a corrosão de ligas Fe-Cr e Fe-Co aspergidas termicamente por arco elétrico
}

\author{
Microstructural and corrosion resistance \\ aspects of Fe-Cr and Fe-Co alloys \\ thermically sprayed by electric arc
}

Erika dos Santos Pereira ${ }^{1}$, Hector Reynaldo Meneses Costa ${ }^{1}$, André Rocha Pimenta ${ }^{2}$, Marilia Garcia Diniz ${ }^{3}$

\begin{abstract}
${ }^{1}$ Centro Federal de Educação Tecnológica Celso Suckow da Fonseca - CEFET-RJ, Programa de Pós Graduação em Engenharia Mecânica e Tecnologia dos Materiais, PPEMM, CEP 20271-204, Rio de Janeiro, RJ, Brasil.

${ }^{2}$ Instituto Federal do Rio de Janeiro - IFRJ, Laboratório de Instrumentação e Simulação Computacional Científicas Aplicadas - LISComp, CEP 26600-00, Paracambi, RJ, Brasil

${ }^{3}$ Universidade do Estado do Rio de Janeiro - UERJ, Departamento de Engenharia Mecânica, Programa de Pós-Graduação em Engenharia Mecânica - PPGEM, CEP 20940-903, Rio de Janeiro, RJ, Brasil.

e-mail: erika.eng.mec@gmail.com, hectorey@gmail.com, andre.pimenta@ifrj.edu.br, diniz@uerj.br
\end{abstract}

\begin{abstract}
RESUMO
Este trabalho teve como objetivo quantificar e avaliar através de técnicas de análise e processamento digital de imagens (PDI) defeitos formados em revestimentos metálicos à base de ligas ferro-cromo e ferro-cobalto, obtidas por aspersão térmica à arco elétrico (AT). Imagens obtidas por microscopia óptica (MO) foram processadas e analisadas utilizando-se o software FIJI (Image J) para quantificação de poros, redes de óxidos e trincas. Também foram feitas medidas de microdureza Vickers (HV) e testes de corrosão segundo a norma ASTM-G48. Foram realizados dois testes de corrosão em meio de cloreto férrico: um expondo-se apenas o revestimento, onde a resistência a corrosão foi avaliada qualitativamente através de imagens de MO e a possível presença de pites de corrosão e outro expondo-se o conjunto revestimento e substrato, neste caso, a resistência a corrosão foi avaliada quantitativamente através de medidas na espessura da camada de revestimento antes e depois da exposição. Foi realizada uma avaliação microestrutural por MO e microscopia eletrônica de varredura (MEV). Os resultados obtidos por MO, MEV e microdureza indicaram uniformidade nas camadas depositadas e porcentagem de defeitos variando entre 3,7\% e 4,3\% para os revestimentos estudados. Todos mostraram possuir excelente resistência à corrosão em meio de cloreto férrico, que é considerado agressivo aos aços inoxidáveis, exceto para a liga de revestimento à base de ferro-cobalto quando encontrouse combinada ao cobre e com o substrato também exposto ao meio corrosivo
\end{abstract}

Palavras-chave: Aspersão térmica. Revestimentos metálicos. Processamento digital de imagens. Teste de corrosão.

\section{ABSTRACT}

The aim of this work was to quantify and evaluate, through techniques of analysis and digital image processing (PDI), defects formed in metallic coatings based on ferro-nickel, iron-chromium and cobaltchromium alloys obtained by thermal arc-electric spraying. Images obtained by optical microscopy (OM) were processed and analyzed using the FIJI software (Image J) for quantification of pores, oxide networks and cracks. Measurements of Vickers microhardness (HV) and corrosion tests according to the ASTM-G48. Two corrosion tests were carried out in ferric chloride medium: one exposing only the coating, where the corrosion resistance was evaluated qualitatively through OM images and the possible presence of corrosion pits and the other exposing the coating and substrate set, in this case, the corrosion resistance was quantitatively evaluated through measurements on the thickness of the coating layer before and after exposure. An assessment of the microstructural morphology by optical microscope (OM) and scanning electron microscope (SEM) was made. The results obtained by OM and SEM indicated that the coatings showed uniformity 
throughout the deposited layer and low number of defects (oxides, cracks and porosities). The percentage of defects found varied between $3.7 \%$ and $4.3 \%$ for the coatings studied. All showed excellent resistance to corrosion in ferric chloride, which is considered aggressive to stainless steels, except for the cobalt-based coating alloy when it was combined with copper and with the substrate also exposed to the corrosive environment.

Keywords: Thermal spraying. Metal coatings. Digital image processing. Corrosion test.

\section{INTRODUÇÃO}

A corrosão de materiais metálicos é um grave problema enfrentado pelas indústrias, muitas vezes, é o mecanismo responsável pela falha de equipamentos [1-3]. Diversas soluções podem ser implementadas com o objetivo de evitar processos de corrosão: utilização de aços inoxidáveis de alta resistência à corrosão [4-6], aplicação de revestimentos inorgânicos [7-9] ou aplicação de revestimentos metálicos [10-12]. A seleção da melhor técnica depende de diversos fatores, tais como o ambiente corrosivo, a geometria da peça, capacidade de aderência ao material do substrato, custos e tempo de vida esperado para o equipamento [13].

A aplicação de revestimentos protetores, com finalidades de engenharia, iniciou-se para proteção de conjuntos e componentes mecânicos contra a corrosão. Com o avanço de pesquisas no desenvolvimento de novos materiais, os revestimentos são utilizados também para elevar a resistência ao desgaste, permitir o isolamento térmico e elétrico e contra incompatibilidades químicas e biológicas e já estão sendo utilizados para implantes. O desenvolvimento da tecnologia de aspersão térmica (AT) para obtenção de revestimentos baseia-se na necessidade de prolongar a vida útil de sistemas e peças, em face o elevado custo de componentes de reposição aliado as vantagens de recuperar peças as quais se elimina a substituição [14, 15].

A AT é uma técnica importante na proteção de materiais contra a corrosão e o desgaste abrasivo; trata-se de um processo que permite o uso de grande variedade de ligas para revestimento, em certos casos de baixo custo e prático, uma vez que é possível o transporte das ferramentas, permitindo que o trabalho seja feito em campo. Atualmente, a utilização da técnica de AT vem aumentando, devido à preocupação por parte das empresas, principalmente da área de óleo e gás, em aumentar a vida útil dos materiais expostos ao ambiente marinho severo em relação à corrosão. Revestimentos obtidos por AT têm por finalidade atender requisitos altamente exigentes e condições de serviços agressivas que muitas vezes podem acarretar acidentes, perda prematura de componentes e paradas de produção [15].

Uma estrutura típica de revestimentos aspergidos é constituída de lamelas do material depositado permeado de inclusões de óxidos, microtrincas, partículas sólidas e porosidade, e tudo isto é considerado como "imperfeições", artefatos deletérios ou defeitos contidos em uma camada de revestimento [14, 16].

A porosidade em revestimentos é uma propriedade importante em relação a resistência à corrosão, uma vez que a proteção do substrato contra o meio agressivo depende, em parte, da existência de poros [17]. Porosidades, trincas e fissuras no revestimento são consideradas falhas que irão gerar uma permeabilidade nociva na camada protetora do substrato. A maior ou menor presença destes defeitos é função de parâmetros do processo de AT [18].

A resistência à corrosão do aço pode ser significativamente aumentada modificando-se a superfície com revestimentos anticorrosivos aspergidos termicamente. Uma redução substancial de custos pode ser obtida em comparação com o uso de ligas convencionais [19]. Estes revestimentos devem apresentar elevada resistência a corrosão, além de boa aderência e a menor quantidade de defeitos possíveis [20].

Testes de corrosão podem ter como objetivo investigar o comportamento do material quando exposto a um determinado meio, ou ser realizado para verificar se o material fornecido está de acordo com o requisito especificado, ou ainda para qualificar um determinado procedimento [21]. A norma ASTM G-48 verifica a corrosão por pites em meio de cloreto férrico [22], sendo um dos testes de controle mais utilizados por indústrias petroquímicas para verificar a qualidade de produtos [23]. A corrosão por pites pode levar a falha do equipamento de forma rápida, sendo importante verificar a susceptibilidade do material a este processo [2427].

Este trabalho utilizou a combinação de diferentes ligas comerciais para criar quatro novos revestimentos para aplicações em AT que demandem alta resistência à corrosão. Quatro ligas à base de ferro-cromo ou ferro-cobalto foram utilizadas. Para verificar as características dos revestimentos produzidos, foi feita a quantificação geral de defeitos (poros, trincas e regiões com redes de óxidos) através de análise e processamento digital de imagens (PDI) e testes de microdureza Vickers ao longo da espessura dos revestimentos. A resistência à corrosão foi avaliada através de testes baseados na norma ASTM G-48, em meio de cloreto férrico, onde foram analisados aspectos qualitativos dos materiais testados, antes e depois do contato com o agente corrosivo. 


\section{MATERIAIS E MÉTODOS}

Quatro diferentes revestimentos foram obtidos à partir da combinação de quatro diferentes arames (Tabela 1). Estes revestimentos foram depositados sobre substrato de aço carbono UNS G10200 através do processo de AT arco elétrico.

Tabela 1: Arames utilizados e composição química (\% em massa).

\begin{tabular}{c|c|c|c|c|c|c|c|l}
\hline ARAME & $\mathbf{F e}$ & $\mathbf{C r}$ & $\mathbf{N i}$ & $\mathbf{M n}$ & $\mathbf{M o}$ & $\mathbf{C}$ & $\mathbf{S i}$ & \multicolumn{1}{c}{ OUTROS } \\
\hline $\mathrm{A}$ & 66,1 & 27,0 & & 1,8 & & & 1,6 & $\mathrm{~B} 3,5$ \\
\hline $\mathrm{B}$ & 65,7 & 25,7 & 2,9 & 1,9 & 0,8 & 1,6 & 1,4 & \\
\hline $\mathrm{C}$ & 3,6 & 28,8 & 1,9 & 0,9 & 0,02 & 1,1 & 0,3 & $\mathrm{Co} 58,4 / \mathrm{W} 4,9$ \\
\hline $\mathrm{D}$ & 68,5 & 19,6 & 9,1 & 1,5 & 0,5 & 0,02 & 0,3 & $\mathrm{Cu} 0,4 / \mathrm{P} 0,03 / \mathrm{N} 0,07 / \mathrm{Nb} 0,01$ \\
\hline
\end{tabular}

A Tabela 2 apresenta as quatro combinações feitas (nomeadas Liga 1, Liga 2, Liga 3 e Liga 4) entre os arames listados na Tabela 1.

Tabela 2: Condições geradas pela combinação de arames para obtenção do revestimento.

\begin{tabular}{c|c}
\hline CONDIÇÃO & COMBINAÇÃO DOS ARAMES \\
\hline 1 & $\mathrm{~A}+\mathrm{B}$ \\
\hline 2 & $\mathrm{~B}+\mathrm{C}$ \\
\hline 3 & $\mathrm{C}+\mathrm{D}$ \\
\hline 4 & $\mathrm{~B}+\mathrm{D}$ \\
\hline
\end{tabular}

Cada uma das ligas utilizadas para o revestimento (Tabela 2) foi aspergida sobre uma chapa de substrato de aço carbono UNS G10200 com as dimensões $100 \mathrm{~mm}$ x $150 \mathrm{~mm}$ x 4,5 mm. Estas chapas passaram, antes de serem revestidas, por processo de limpeza mecânica por jateamento abrasivo com óxido de alumínio G.20, obtendo-se uma superfície grau Sa $2^{\wedge}(1 / 2)$ segundo a norma ISO 5801, o que resultou em uma rugosidade média Ra de $179 \mu \mathrm{m}$. A deposição dos arames, que possuíam 2,6 mm de diâmetro, foi realizada até obtenção de espessura não maior que $2 \mathrm{~mm}$ de revestimento. Os seguintes parâmetros foram utilizados: tensão de $40 \mathrm{~V}$; corrente aproximada de $100 \mathrm{~A}$; taxa de deposição de $3,24 \mathrm{~kg} / \mathrm{h}$; ângulo entre a pistola e o substrato de $90^{\circ}$, e ar comprimido como gás de proteção.

Amostras das chapas revestidas foram obtidas por cortes transversais em cortadeira metalográfica e com discos de dureza superior a 45HRc. Em seguida, elas foram embutidas em resina de baquelite, lixadas e polidas até a obtenção de superfícies especulares. As amostras foram numeradas conforme a condição (Tabela 2). Imagens de microscopia ótica (MO) para seções transversais dos revestimentos foram obtidas através de um microscópio Olympus BX60M. Dez imagens de áreas não superpostas (500x) de cada condição foram submetidas a etapas de PDI através do programa FIJI Image J (https://imagej.net/Fiji/Downloads). Foram realizados procedimentos para a identificação e quantificação do percentual de poros, trincas e redes de óxidos.

Medidas de dureza Vickers (HV) foram realizadas por um Microdurômetro WILSON INSTRUMENTS, modelo 422 MVD. A carga aplicada foi de 0,3 kgf e o tempo de aplicação de carga de cinco segundos. Foram selecionados 8 pontos randômicos em diferentes regiões de cada um dos revestimentos testados.

Os testes de corrosão foram baseados na norma ASTM-G48, destinada a ensaios de corrosão por pits de aços inoxidáveis. Não foi possível a aplicação da norma tal como descrita, pois a mesma não é definida para materiais revestidos e sim para materiais homogêneos. Amostras das quatro condições foram embutidas em baquelite de forma a deixar apenas o revestimento exposto ao ataque, e então, cada uma delas foi imersa em $600 \mathrm{~mL}$ de solução de cloreto férrico a $6 \%$ por 72 horas em temperatura ambiente. A resistência à corrosão foi avaliada pela formação de pits de corrosão, utilizando imagens de MO (20 x magnificação), conforme previsto na norma ASTM-G48.

A ocorrência de um dano ao revestimento (descolamentos, riscos etc.), embora indesejável, pode ocorrer em materiais revestidos, fato que pode desencadear um processo de corrosão mais agressivo. Com o objetivo de simular essa situação, o mesmo procedimento para teste de corrosão relatado anteriormente foi realizado em amostras sem embutimento, de forma que tanto o revestimento como o substrato ficaram expostos 
ao meio corrosivo. A resistência à corrosão nesta situação foi aferida pela medição da espessura da camada de revestimento antes e após o teste de corrosão.

Os revestimentos estudados foram submetidos à análise química semi-quantitativa com $0,3 \%$ de precisão, por técnica de Energy Dispersive Spectroscopy (EDS) através de um dispositivo acoplado a um microscópio eletrônico de varredura (MEV) marca Bruker QUANTAX EDS com detectores XFlash ${ }^{\circledR}$ 6, com o intuito de comparação entre os elementos químicos presentes nos arames e os elementos químicos incorporados nas camadas aspergidas.

\section{RESULTADOS E DISCUSSÃO}

A Figura 1 exemplifica os aspectos obtidos por MO para cada uma das condições testadas neste trabalho, onde foram evidenciados defeitos típicos do processo de aspersão técnica e uma estrutura de camadas superpostas tipicamente lamelar [18]. A técnica de PDI se baseia na identificação pelo computador de objetos de interesse a serem quantificados e já está consagrada para avaliação de defeitos em camadas revestidas por AT [28]. Os defeitos apresentaram tonalidades mais escuras que o material do revestimento, permitindo assim a identificação dessas regiões pelo computador. A Figura 1 apresenta exemplos dos aspectos microestruturais obtidos por MO e identificação de defeitos. A Figura 2 exemplifica região de revestimento usada na identificação dos defeitos e após quantificação por PDI. A Tabela 3 apresenta os resultados obtidos para a fração volumétrica (Vv) de defeitos para todos os revestimentos estudados.

Tabela 3: Fração volumétrica de defeitos (Vv) para as condições testadas.

\begin{tabular}{c|c|c}
\hline \multirow{2}{*}{ CONDIÇÃo } & \multicolumn{2}{|c}{ Vv (\%) } \\
\cline { 2 - 3 } & Média & Desvio Padrão \\
\hline Liga 1 & 3,7 & 2,6 \\
\hline Liga 2 & 4,3 & 2,3 \\
\hline Liga 3 & 2,2 & 1,4 \\
\hline Liga 4 & 1,4 & 0,6 \\
\hline
\end{tabular}

Todas as situações (Figura 1) mostram camadas superpostas, conhecidas como lamelas (típicas do processo de AT), regiões com tonalidades mais escuras isoladas ou agrupadas que podem ser pequenos poros ou redes de óxidos, bem como a presença de partículas não fundidas durante a deposição, com morfologia característica esférica, dentro do padrão gerado por AT [14, 29].

As ligas 1 e 2 foram as que apresentaram maior fração volumétrica de defeitos. Ambas eram compostas por combinações do arame b com os arames a e c, resultando sempre em ligas com alto teor de cromo. Já as ligas 3 e 4 são compostas por combinações utilizando o arame d, que possui menor teor de cromo. A condição 4 foi a de menor percentual de defeitos, esta composta pelos arames d e b, destacando-se pela presença de níquel. A condição 2, que também teve um baixo percentual de defeitos, foi formada pela combinação dos arames c e d, com valor expressivo de cobalto e com a presença de níquel. A presença de poros não costuma estar associada à composição química dos arames usados na AT por arco elétrico, entretanto, as redes de óxidos sim. Uma maior quantidade de redes de óxidos irá significar uma maior Vv de defeitos [14].

A presença de poros é comum em todos os processos de AT, resultante das condições adversas que as partículas sofrem durante a pulverização e ao impacto. A porosidade depende basicamente da velocidade da partícula, ângulo de pulverização, temperatura e tamanho das partículas. Algumas dessas não são fundidas, contribuindo na criação de espaços vazios, formando os poros. Poros e trincas são defeitos que podem diminuir a resistência à corrosão do sistema revestimento / substrato [30].

A Tabela 4 mostra os resultados de microdureza obtidos para todas as condições testadas (Ligas 1, 2, 3, e 4). Observa-se que a condição com maior dureza foi a liga 1 (liga Fe-Cr-Ni-Mn-Mo). 


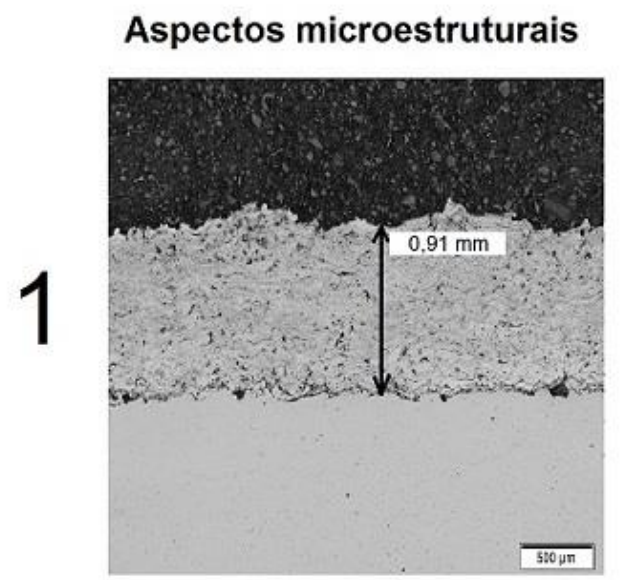

\section{Identificação de defeitos}
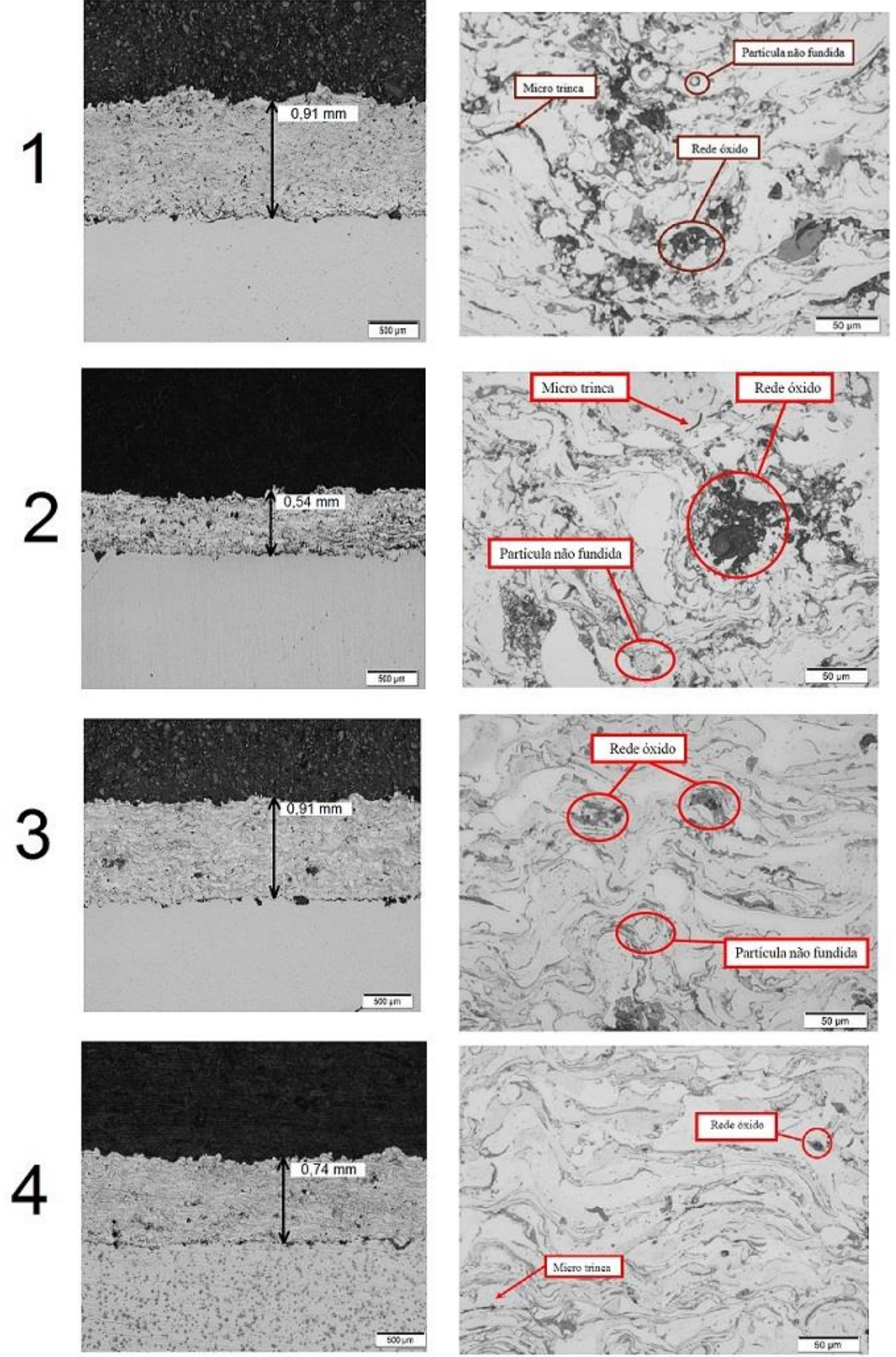

Figura 1: Imagens em MO dos revestimentos para todas as condições estudadas (Ligas 1, 2, 3 e 4) 

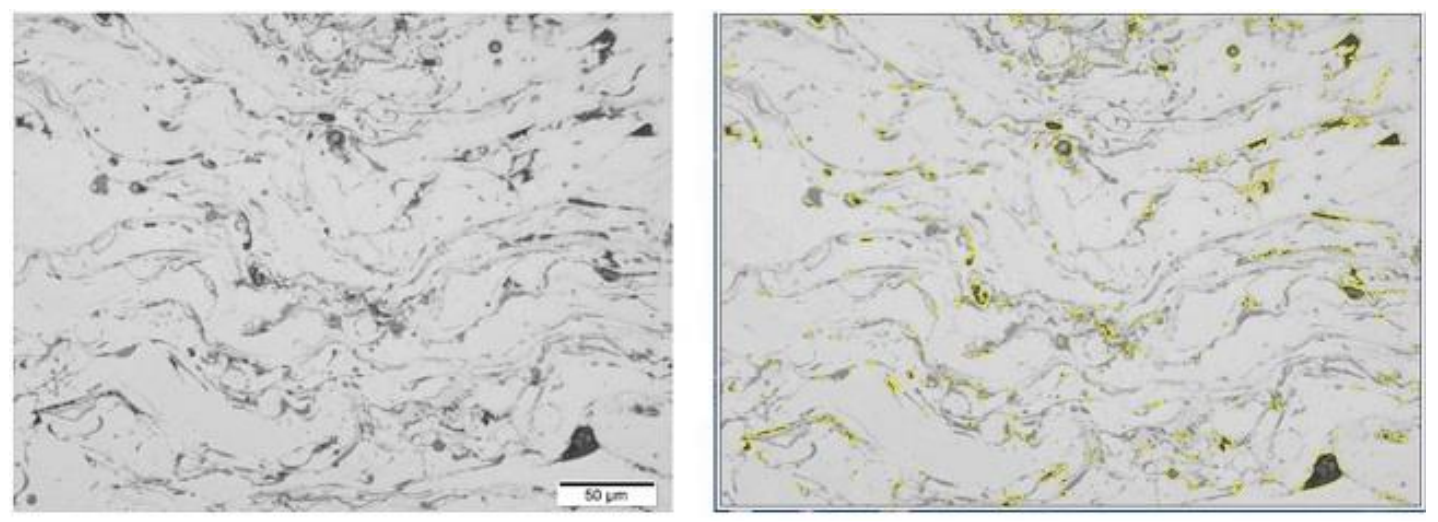

Figura 2: Região do revestimento estudada (esquerda) e defeitos quantificados por PDI (direita)

Tabela 4: Resultados de microdureza

\begin{tabular}{c|c|c|c|c}
\hline HV & 1 & 2 & 3 & 4 \\
\hline Média & 592,5 & 330,8 & 297,0 & 324,4 \\
\hline DP & 127,0 & 80,1 & 77,4 & 67,8 \\
\hline
\end{tabular}

Foram obtidos valores de desvio padrão (DP) mais elevados nas medidas realizadas nas condições $1 \mathrm{e}$ 2, ambas com as maiores quantidades percentuais de defeitos medidos (3,7 e 4,3\%, respectivamente). Assim, a presença maior de defeitos parece ter provocado uma maior dispersão das medidas e nos respectivos desvios padrão. A dureza é essencialmente afetada pela composição química da liga, mas os defeitos afetam a dispersão das medidas.

O processo AT normalmente mantém os valores de dureza entre 345 a $392 \mathrm{HV}$. Outros processos, tal como o AT por HVOF (Chama de Oxi-gás de Alta Velocidade), consegue fornecer valores ainda maiores, normalmente entre 595 a $1354 \mathrm{HV}$ para materiais não ferrosos [31].

A Figura 3 apresenta imagens que mostram aspectos superficiais dos revestimentos antes e depois do teste de corrosão realizado com as amostras embutidas em baquelite, onde apenas o revestimento foi posto em contato com o agente corrosivo, neste caso solução de cloreto férrico $6 \%$ (v/v). É possível observar que os aspectos antes e depois, em todas as situações, foi muito parecido, não foram notados resíduos ou tonalidades de detritos que pudessem estar associados aos produtos de corrosão dos revestimentos ou substrato.

A Tabela 5 apresenta as espessuras dos revestimentos antes e depois dos testes de corrosão para as amostras não embutidas, isto é, amostras cujo sistema substrato de aço carbono / revestimento foram imersas em meio cloreto férrico. Pequenas diferenças entre a espessura antes do teste de corrosão e depois do teste de corrosão, tal como para as condições 1, 2 e 4, estão relacionadas à rugosidade ou topografia irregular das superfícies, que é comum das superfícies geradas por AT, uma vez que estas medidas não puderam ser aferidas em muitos locais [18]. Apenas uma medida foi realizada para cada condição.

Tabela 5: Espessuras (valores em mm) dos revestimentos antes e depois do ensaio de corrosão

\begin{tabular}{c|c|c}
\hline LIGAS & ESPESSURA INICIAL & ESPESSURA FINAL \\
\hline 1 & 0,91 & 0,85 \\
\hline 2 & 0,54 & 0,55 \\
\hline 3 & 0,91 & ----- \\
\hline 4 & 0,74 & 0,77 \\
\hline
\end{tabular}




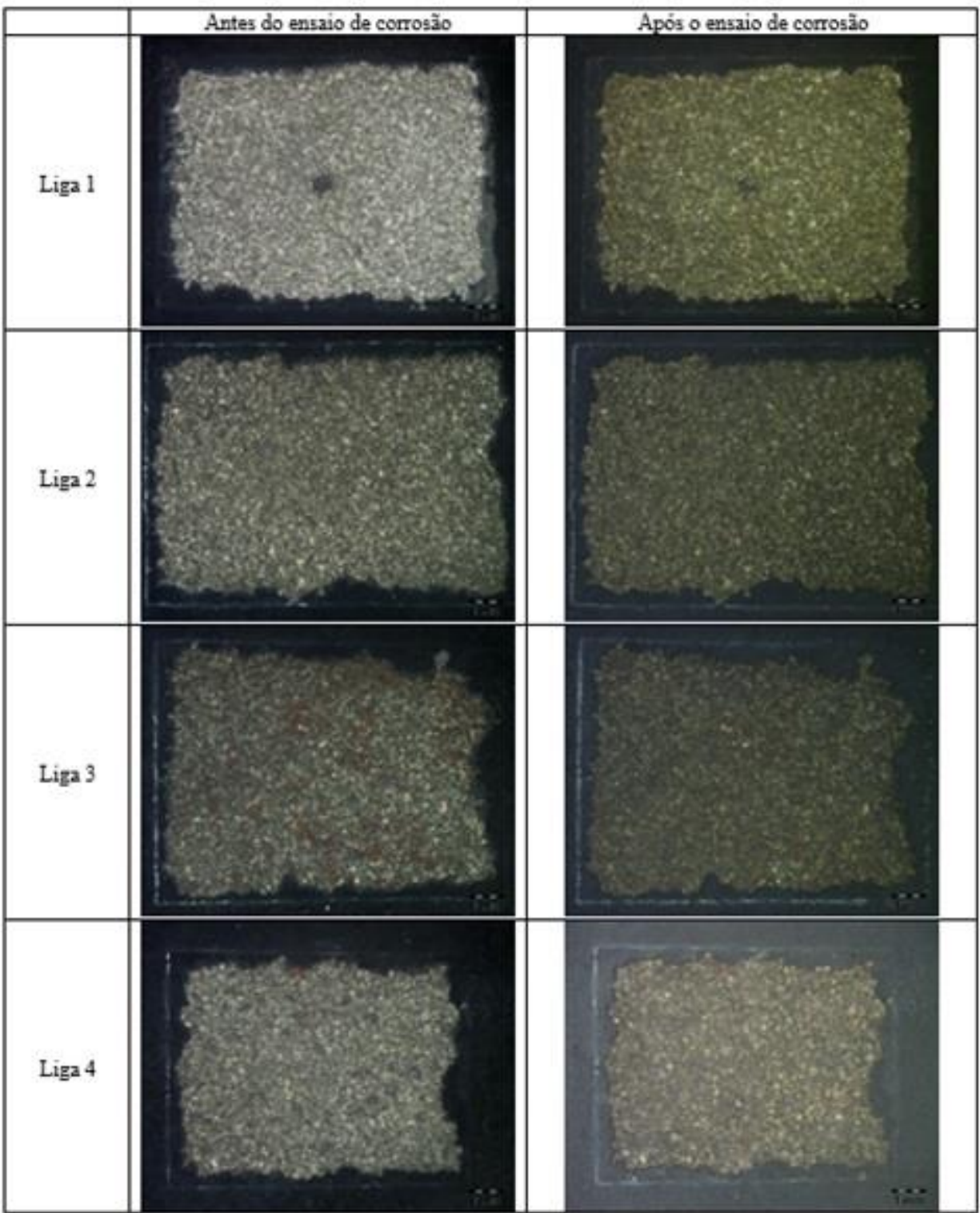

Figura 3: Aspectos das superfícies revestidas antes x depois dos testes de corrosão em cloreto férrico 6\%

Apesar das medidas únicas, pode-se dizer que as condições 1, 2 e 4 não tiveram suas espessuras de revestimento afetadas pela corrosão em meio cloreto. Isso pode ser observado nas Figuras 4 e 5 . Na Figura 4 são apresentadas as imagens obtidas em MEV dos revestimentos após o ensaio de corrosão das ligas 1, 2 e 4, onde não foi observado um processo de corrosão acentuado nos mesmos. A Figura 5 apresentada uma imagem em MO do revestimento da liga 4 após o teste de corrosão, exemplificando as medidas de espessura realizadas.

Uma diminuição de massa das amostras não embutidas foi percebida, porém, essencialmente proporcionada pela corrosão / deterioração do substrato e não pela corrosão dos revestimentos, que permaneceram praticamente com as mesmas espessuras e aspectos, com exceção do revestimento da condição 3. Este último foi totalmente corroído pelo reagente químico e, portanto, sua espessura final não pôde ser medida (Tabela 5). A condição 3, única onde a presença de cobre no revestimento era significativa, mostrou-se sensível ao meio no qual o substrato também estava exposto em conjunto. Assim, pôde-se concluir que o sistema revestimento / substrato, ambos em contato com o reagente, foi propício ao processo corrosivo do revestimento, o que não ocorreu quando apenas o revestimento foi exposto ao meio (amostra embutida). 


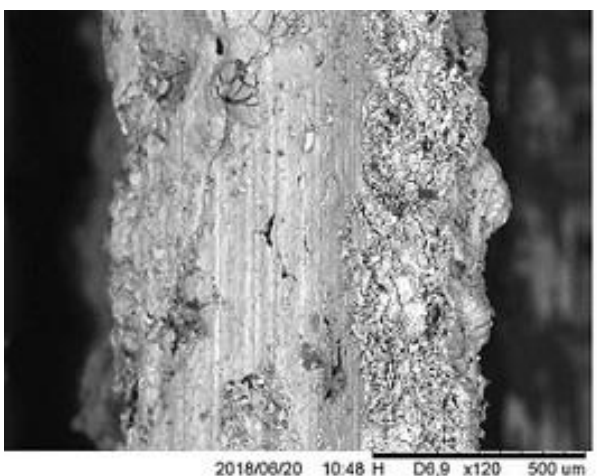

(a)

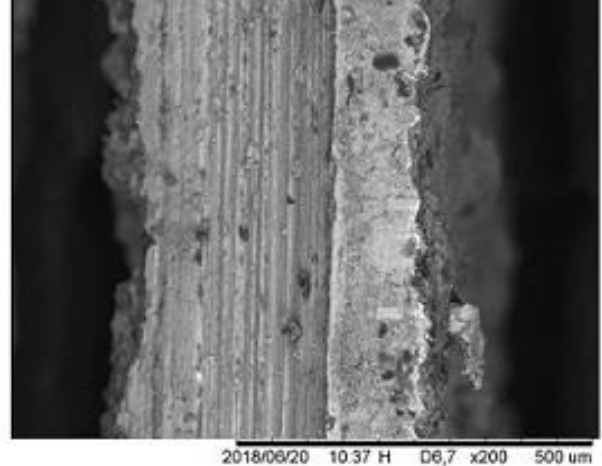

(b)

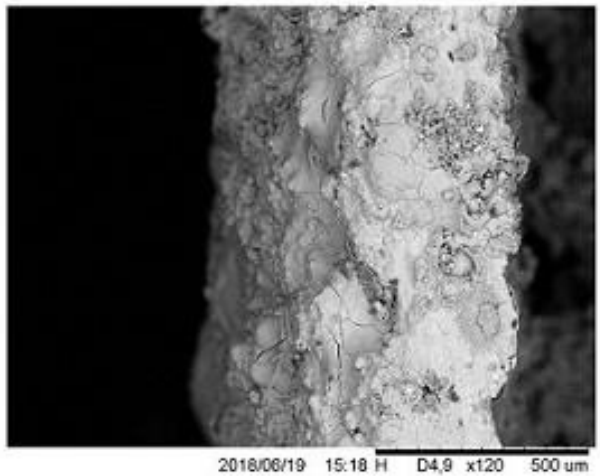

(c)

Figura 4: Aspectos da seção transversal das condições 1 (a), 2 (b) e 4 (c) de amostras não embutidas após o ensaio de corrosão. MEV.

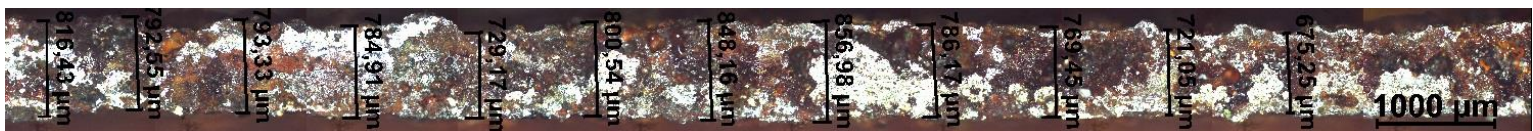

Figura 5: Mosaico obtido para seção transversal do revestimento para a condição 4 da amostra não embutida. As medidas de espessura da camada aspergida variam no entorno de $0,77 \mathrm{~mm}$

A utilização do ensaio de corrosão por pits de aços inoxidáveis para revestimentos obtidos por aspersão térmica mostra-se uma boa alternativa em relação aos ensaios mais complexos, como o ensaio de névoa salina e de polaridade [18].

Os resultados de EDS mostraram que os elementos químicos presentes em maiores quantidades nas combinações de arames foram essencialmente incorporados aos revestimentos, isto é, o processo de aspersão não determinou a perda dos elementos principais dos arames originalmente usados. A Figura 6 apresenta apenas o resultado da condição 2, entretanto, todas as condições analisadas estão em consonância.

Esta influência da combinação dos elementos de liga na resistência à corrosão em revestimentos aspergidos termicamente já foi reportado por alguns trabalhos [19, 31-35]. Esses autores verificaram também, que a presença dos defeitos (trincas, poros e redes de óxidos) comprometeram a resistência à corrosão dos revestimentos, mostrando a importância dos ajustes dos parâmetros operacionais de aspersão e a combinação de elementos de liga, quando se deseja associar resistência mecânica e resistência à corrosão. Contudo, no presente trabalho não foi identificada uma relação entre a quantidade de defeitos e a resistência a corrosão. Atribui-se tal comportamento ao fato de todos as amostras possuírem baixa fração volumétrica de defeitos. A liga 2 foi a condição com maior quantidade de defeitos, apresentando 4,3\%. Em ligas fabricadas pelo processo de arco elétrico são esperados que óxidos representes entre 1 e $3 \%$ da fração volumétrica, e a porosidade entre a 2 e $7 \%$, portanto, defeitos entre 3 e $10 \%$ são esperados. A corrosão sofrida pela liga 3, não está associada a quantidade de defeitos, mas possivelmente a um processo de corrosão galvânica, formada pela exposição em conjunto do revestimento e do substrato, pois quando o substrato foi protegido com a baquelite e apenas o revestimento exposto ao meio corrosivo, a liga não sofreu processo de corrosão. 

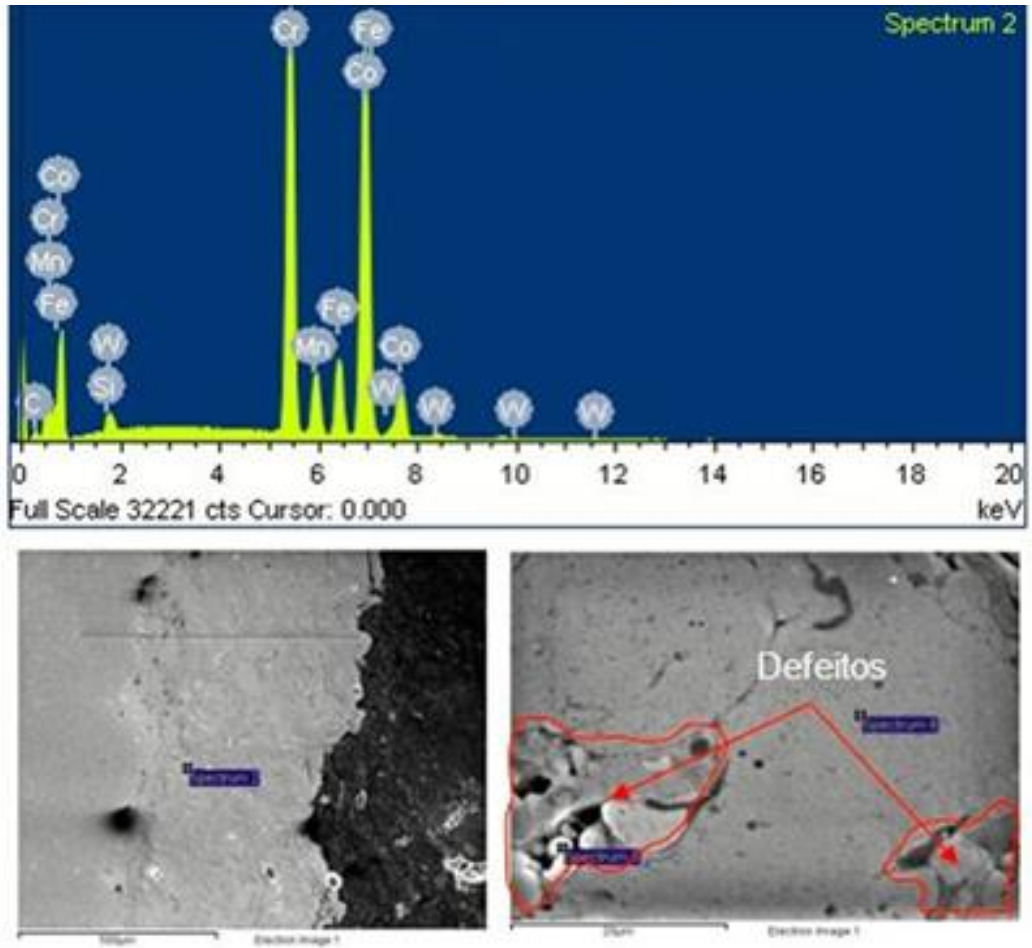

Figura 6: Espectro de energias características obtido por EDS para a região revestida na condição 2. Foram identificadas as presenças dos elementos em maiores quantidades: Fe, Co e Cr. A presença de defeitos (poros e redes de óxidos) foi confirmada

\section{CONCLUSÕES}

Analisando os resultados obtidos, pôde-se concluir que:

1. Os revestimentos apresentaram-se com estrutura lamelar tipicamente associada ao processo de aspersão térmica e os elementos presentes em maiores quantidades nos arames foram incorporados aos revestimentos.

2. A presença de óxidos, poros e trincas aparentemente não diminuiu a dureza dos revestimentos, mas provocaram um aumento na dispersão das medidas. As condições com maiores desvios foram a 1 (liga $\mathrm{Fe}$ Cr-B-Mn) e 2 (liga Fe-Co-Cr-Ni).

3. Todos os revestimentos e sistemas testados tiveram excelente desempenho para os revestimentos frente ao reagente agressor cloreto férrico, exceto o sistema revestimento / substrato exposto / condição 3 . Esta condição era a única que tinha a presença de cobre como componente de liga. $\mathrm{O}$ revestimento continha ferro, cobalto, cromo e níquel com uma pequena fração de cobre, fósforo, nitrogênio e nióbio e não reagiu bem ao ambiente corrosivo em estudo, apesar de possuir apenas 2,2\% de fração volumétrica de defeitos. As frações volumétricas de defeitos variaram entre 1,4\% - 4,3\%. Esses valores foram considerados satisfatórios para revestimentos obtidos por técnica de AT arco elétrico.

\section{AGRADECIMENTOS}

Os autores agradecem ao CNPq pelo suporte financeiro e pela bolsa de estudo. Esse estudo foi financiado em parte pela Coordenação de Aperfeiçoamento de Pessoal de Nível Superior - Brasil (CAPES) - Código de Financiamento 001. Também agradecem a empresa VGK e ao seu Diretor Técnico Guilherme W. Bungner.

\section{BIBLIOGRAFIA}

[1] WANG, Z., ZHOU, Z., XU, W., et al., "Study on inner corrosion behavior of high strength product oil pipelines", Engineering Failure Analysis, v. 115, pp. 104659, Set. 2020. 
[2] CHANDRA, K., KAIN, V., SINHA, S.K., et al., "Metallurgical investigation of a heat-exchanger tube of 70/30 cupronickel failed by fretting corrosion", Engineering Failure Analysis, v. 116, pp. 104756, Oct. 2020.

[3] RESS, J., MONRRABAL, G., DÍAS, A., et al., "Microbiologically influenced corrosion of welded AISI 304 stainless steel pipe in well water", Engineering Failure Analysis, v. 116, pp. 104734, Oct. 2020.

[4] PIMENTA, A.R., DINIZ, M.G., PEREZ, G., et al., "Nitrogen addition to the shielding gas for welding hyper-duplex stainless steel", Soldagem e Inspeção, v. 25, pp. 1-8, Jun. 2020.

[5] FONSECA, G.S., BARBOSA, L.O.R., FERREIRA, E.A., et al., "Microstructural, mechanical, and electrochemical analysis of duplex and superduplex stainless steels welded with the autogenous TIG process using different heat input", Metals, v. 7, n. 12, pp. 538, Dec. 2017.

[6] FONSECA, G.S., OLIVEIRA, P.M., DINIZ, M.G., et al., "Sigma phase in superduplex stainless steels: formation, kinetics and microstructural path", Materials Research, v. 20, n. 1, pp. 249-255, 2017.

[7] BRAGA, A.V.C., LAGO, D.C.B., PIMENTA, A.R., et al., "The influence of heat treatment of inorganic conversion coatings produced by sol-gel dip coating on the anticorrosive properties of alumina films deposited on steel substrate - Part I: Single conversion coatings", Surface and Coatings Technology, v. 372, pp. 190200, May. 2019.

[8] BRAGA, A.V.C., LAGO, D.C.B., PIMENTA, A.R., et al., "The influence of heat treatment of inorganic conversion coatings produced by sol-gel dip coating on the anticorrosive properties of alumina films deposited on steel substrate - Part II: silica/boehmite or boehmite/silica multilayered conversion coatings", Surface and Coatings Technology, v. 386, pp. 125500, Feb. 2020.

[9] TIWARI, S.K., SAHU, R.K., PRAMANICK, A.K., et al., "Development of conversion coating on mild steel prior to sol gel nanostructured $\mathrm{Al} 2 \mathrm{O} 3$ coating for enhancement of corrosion resistance", Surface and Coatings Technology, v. 205, pp. 4960-4967, 2011.

[10] CAMPOS, P.K., CARDOSO, B.R., FURTADO, H.C., et al., "Characterization of mechanical and surface finishing properties of metallic coating obtained by arc electric thermal spray", Brazilian Journal of Development, v. 6, n. 3, pp. 11078-11090, mar. 2020.

[11] SZYMANSKI, K., HERNAS, A., MOSKAL, G., et al., "Thermally sprayed coatings resistant to erosion and corrosion for power plant boilers - a review", Surface and Coatings Technology, v. 268, pp. 153-164, Apr. 2015.

[12] LEE, H.S., SINGH, J.K., ISMAIL, M.A. et al., "Corrosion mechanism and kinetics of Al-Zn coating deposited by arc thermal spraying process in saline solution at prolong exposure periods", Scientific Reports, v. 9, pp. 3399, 2019.

[13] ASM, “ASM handbook, vol. 13 corrosion", 9 th., Ohio, ASM International, 1987.

[14] PAREDES, R.S.C., "Aspersão Térmica, em projeto de laboratório de aspersão térmica e soldagem especiais”, Dissertação de M.Sc., Universidade Federal do Paraná, Curitiba, PR, Brasil, 2012.

[15] FREITAS, B.C., "Estudo das propriedades microestruturais de ligas de níquel-cromo e cobalto-cromo obtidas pelo processo de metalização a arco", Dissertação de M.Sc., CEFET/RJ, Rio de Janeiro, RJ, Brasil, 2015.

[16] SILVA, V.C., PAREDES R.S.C., "Avaliação da relação $\mathrm{Cr}_{\text {eq. }} / \mathrm{Ni}_{\text {eq. }}$ para o revestimento AF2209 depositado por aspersão térmica com e sem pré-aquecimento", Revista Matéria, v. 21, n.2, pp. 470-481, 2016.

[17] VREIJLING, M.P.W., "Electrochemical characterization of metallic thermally sprayed coatings". Ph.DThesis, TNO Institute of industrial Tecnology, Netherlands,1998.

[18] BRITO, V.R.S., "Caracterização de revestimento metálicos aspergidos termicamente por arco elétrico", Dissertação de M.Sc., CEFET/RJ, Rio de Janeiro, RJ, Brasil, 2010.

[19] PARK, IL-CHO, KIM, SEONG-JONG," Corrosion behavior in seawater of arc thermal sprayed Inconel 625 coatings with sealing treatment”, Surface \& Coatings Technology, v. 325, pp. 729-737, 2017.

[20] AGUILA, J.V., BUSCHINELLI, A.J.A., PAREDES, R.S.C., "Caracterização de consumíveis antidesgaste e anticorrosão produzidos especialmente para aspersão térmica por arco elétrico e utilizados para revestir ponteiras de riser para plataformas offshore”, Revista Iberoamericana de Ingeniería Mecánica., v. 13, n. 1, pp. 39-49, 2009.

[21] HOLMBERG, B., BERGQUIST, A., "Suitable corrosion test methods for stainless steel welds", Welding in the word, v. 52, n. 2/4, pp. 17-21, 2008.

[22] ASTM, "G 48 - Standard test methods for pitting and crevice corrosion resistance of stainless ssteels and related alloys by use of ferric chloride solution", ASTM American Society for Testing and Materials, 1999. 
[23] Steinsmo, U., Rogne, T., Drugli, J., “Aspects of testing and selecting stainless steels for seawater applications”, Corrosion, v. 53, n. 12, pp. 955-964, Dec. 1997.

[24] MORI, G., BAUERNFEIND, D. "Pitting and crevice corrosion of superaustenitic stainless steels", $M a-$ terials and Corrosion, v. 55, n. 3, pp. 164-173, Mar. 2004.

[25] HOSEINPOORA, M., MOMENIA, M., MOAYEDA, M.H., et al., "EIS assessment of critical pitting temperature of 2205 duplex stainless steel in acidified ferric chloride solution", Corrosion Science, vol. 80, pp. 197-204, Mar. 2014.

[26] PARDO, A., MERINO, M.C., CARBONERAS, A.E., et al., "Pitting corrosion behaviour of austenitic stainless steels with $\mathrm{Cu}$ and Sn additions”, Corrosion Science, vol. 49, n.2, pp. 510-525, Feb. 2007.

[27] YANG, Q., QIAO, L.J., CHIOVELLI, S., et al., "Effects of Hydrogen on Pitting Susceptibility of Type 310 Stainless Steel”, Corrosion, v. 54, n. 8, pp. 628-633, Aug. 1998.

[28] DU, H., SHIN, J. H., WOHN, S., "Study on porosity of plasma-sprayed coatings by digital image analysis method"; Journal of Thermal Spray Technology, v. 14 (4), pp. 453-461, 2005.

[29] VACKEL, A., SAMPATH, S., "Fatigue behavior of thermal sprayed WC-CoCr steel systems: Role of process and deposition parameters", Surface \& Coatings Technology, v. 315, pp. 408-416, 2017.

[30] ŠIMUNOVIĆ, K., Thermal Spraying Welding Engineering and Technology: Thermal Spraying, pp. 1 25. London: Ed. Eolss Publishers, 2010.

[31] MURARIU, A. C., PLEŞU N., PERIANU, I. A., et al., "Investigations on Corrosion Behaviour of WCCrC-Ni Coatings Deposited by HVOF Thermal Spraying Process", International Journal of Electrochemical Science, v. 12, pp. 1535 - 1549, 2017.

[32] MOTTA, F.P., "Propriedades de revestimentos de nióbio obtidos por aspersão térmica a plasma sobre aço API 5L X65", Dissertação de M.Sc., Universidade Federal do Rio Grande do Sul, Porto Alegre, RS, Brasil, 2011.

[33] SONG, B., BAI, M., VOISEY, K.T., et al., "Role of Oxides and Porosity on High-Temperature Oxidation of Liquid-Fueled HVOF Thermal-Sprayed Ni50Cr Coatings", Journal of Thermal Spray Technology, v. 26, pp.554-568, 2017.

[34] BAI, M., REDDY, L., HUSSAIN, T., "Experimental and thermodynamic investigations on the chlorineinduced corrosion of HVOF thermal sprayed NiAl coatings and 304 stainless steels at $700{ }^{\circ}$ C", Corrosion Science, v. 135, pp. 147-157, 2018.

[35] FALS, H. C., ROCA, A. S., FOGAGNOLO, J. B., et al., "Erosion-Corrosion Resistance of Laser Surface Alloying of NbC Thermal Spray Coatings on AISI 304L Steel", Journal of Thermal Spray Technology, v. 29, pp. 319-329, 2020.

\section{ORCID}

Erika dos Santos Pereira

https://orcid.org/0000-0002-1294-3757

Hector Reynaldo Meneses Costa https://orcid.org/0000-0002-0887-6838

André Rocha Pimenta https://orcid.org/0000-0002-5492-3009

Marília Garcia Diniz

https://orcid.org/0000-0002-1836-8618 